\title{
AN INTERIOR INVERSE PROBLEM FOR STURM-LIOUVILLE OPERATORS WITH EIGENPARAMETER DEPENDENT BOUNDARY CONDITIONS
}

\author{
YU PING WANG
}

\begin{abstract}
In this paper, we consider the inverse problem for Sturm-Liouville operators with eigenparameter dependent boundary conditions and show that the potential $q(x)$ and coefficients $\frac{a_{1} \lambda+b_{1}}{c_{1} \lambda+d_{1}}$ and $\frac{a_{2} \lambda+b_{2}}{c_{2} \lambda+d_{2}}$ of the eigenparameter dependent boundary conditions can be uniquely determined by a set of values of eigenfunctions at some interior point and parts of two spectra.
\end{abstract}

\section{Introduction}

In 1929, Ambartsumyan [1] firstly considered the inverse problem for Sturm-Liouville problems. Since 1929, one of this kind of inverse problems was discussed by many authors (see [1], [7]-[18]). Mochizuki and Trooshin [12] studied the inverse problem for interior spectral data of Sturm-Liouville operators on the finite interval $[0,1]$ and showed that a set of values of eigenfunctions at some interior point and parts of two spectra can uniquely determine the potential $q(x)$. Yang (C. F.) and Yang (X. P.) [13] discussed the inverse problem for SturmLiouville operators with discontinuous boundary conditions and proved that the spectral data of parts of two spectra and some information on eigenfunctions at some interior point of the interval $(0, \pi)$ is sufficient to determine the potential $q(x)$.

Consider the following Sturm-Liouville operator $L$ satisfying (1.1)-(1.3)

$$
L y=-y^{\prime \prime}+q(x) y=\lambda y,
$$

with boundary conditions

$$
\left(a_{1} \lambda+b_{1}\right) y(0, \lambda)-\left(c_{1} \lambda+d_{1}\right) y^{\prime}(0, \lambda)=0
$$

or

$$
y^{\prime}(0, \lambda)=h y(0, \lambda) \text { or } y(0, \lambda)=0,
$$

2000 Mathematics Subject Classification. 34A55; 34B24; 47E05.

Key words and phrases. Inverse problem, Sturm-Liouville operator, spectrum, eigenparameter boundary condition. 
and

$$
\left(a_{2} \lambda+b_{2}\right) y(\pi, \lambda)-\left(c_{2} \lambda+d_{2}\right) y^{\prime}(\pi, \lambda)=0 .
$$

where $a_{k}, b_{k}, c_{k}, d_{k} \in \mathbf{R},(-1)^{k} \delta_{k}=a_{k} d_{k}-b_{k} c_{k}<0(k=1,2), q(x)$ is a real-valued function and in $L^{2}[0, \pi]$.

Sturm-Liouville problem with eigenparameter dependent boundary conditions is interesting in Engineering, Physics, Mathematics, etc (see [2]-[10]). In 1977, Fulton [6] studied the Sturm-Liouville problem (1.1), (1.2'), (1.3) and obtained the spectral theory of this kind of problems. Binding, Browne and Seddighi [7] considered the Sturm-Liouville problem (1.1)(1.3) and got the oscillation, comparison results and asymptotic estimates. Using nodal points as spectral data, Browne and Sleeman [8] discussed the inverse nodal problem for the boundary value problem $(1.1),\left(1.2^{\prime}\right),(1.3)$ and showed that a dense set of nodal points of eigenfunctions for the boundary value problem $(1.1),\left(1.2^{\prime}\right),(1.3)$ is sufficient to determine the potential $q(x)$ and coefficient $h$ of the boundary condition. Guliyev [9] found the regularized trace formula for the Sturm-Liouville problem (1.1)-(1.3). Wang, Yang and Huang [10] discussed the half inverse problem for the Sturm-Liouville problem (1.1)-(1.3) and showed that if $q(x)$ is prescribed on $[\pi / 2, \pi]$ and $q-\tilde{q} \in W_{2}^{6}[0, \pi]$, then the potential $q(x)$ on the whole interval $[0, \pi]$ and coefficient $\frac{a_{1} \lambda+b_{1}}{c_{1} \lambda+d_{1}}$ of the eigenparameter dependent boundary condition are uniquely determined by one spectrum.

In this paper, using Mochizuki and Trooshin's method, we discuss the interior inverse problem for Sturm-Liouville problem (1.1)-(1.3) and show that some information on eigenfunctions at some interior point of the interval $(0, \pi)$ and parts of two spectra are sufficient to determine the potential $q(x)$ and coefficients $\frac{a_{1} \lambda+b_{1}}{c_{1} \lambda+d_{1}}$ and $\frac{a_{2} \lambda+b_{2}}{c_{2} \lambda+d_{2}}$ of the eigenparameter dependent boundary conditions.

The following two lemmas are important for us to show the main theorems.

Lemma 1.1. ([7, Theorem 4.2], $[9$, Theorem $])$ Let $\lambda_{n}(n=0,1,2, \ldots)$ be spectrum of the SturmLiouville problem (1.1)-(1.3), then $\lambda_{n}$ is real and simple and satisfies

$$
\begin{aligned}
& \lambda_{0}<\lambda_{1}<\lambda_{2}<\cdots \rightarrow+\infty, \\
& \sqrt{\lambda}_{n}=n\left(1+O\left(\frac{1}{n}\right)\right) .
\end{aligned}
$$

Suppose that $\varphi(x), \theta(x)$ are two fundamental solutions of the equation (1.1) satisfying

$$
\varphi(0)=1, \varphi^{\prime}(0)=0, \theta(0)=0 \text { and } \theta^{\prime}(0)=1,
$$

respectively, then solution of the equation (1.1) satisfying (1.2) is

$$
y(x, \lambda)=\left(c_{1} \lambda+d_{1}\right) \varphi(x)+\left(a_{1} \lambda+b_{1}\right) \theta(x) .
$$

By transformation, we have 
Lemma 1.2. Solution of the equation (1.1) satisfying (1.2) is

$$
\begin{aligned}
y(x, \lambda)= & \left(c_{1} \lambda+d_{1}\right)\left[\cos \sqrt{\lambda} x+\int_{0}^{x} A(x, t) \cos \sqrt{\lambda} t d t\right] \\
& +\left(a_{1} \lambda+b_{1}\right)\left[\frac{\sin \sqrt{\lambda} x}{\sqrt{\lambda}}+\frac{1}{\sqrt{\lambda}} \int_{0}^{x} B(x, t) \sin \sqrt{\lambda} t d t\right],
\end{aligned}
$$

where the kernel $A(x, t)$ satisfies

$$
\frac{\partial^{2} A(x, t)}{\partial x^{2}}-q(x) A(x, t)=\frac{\partial^{2} A(x, t)}{\partial t^{2}}
$$

where $q(x)=2 \frac{d}{d x} A(x, x), A(0,0)=h,\left.\frac{\partial A(x, t)}{\partial t}\right|_{t=0}=0$. The kernel $B(x, t)$ satisfies

$$
\frac{\partial^{2} B(x, t)}{\partial x^{2}}-q(x) B(x, t)=\frac{\partial^{2} B(x, t)}{\partial t^{2}}
$$

where $q(x)=2 \frac{d}{d x} B(x, x), B(x, 0)=0$.

\section{Main results}

Consider another Sturm-Liouville operator $\tilde{L}$

$$
\tilde{L} y=-y^{\prime \prime}+\tilde{q}(x) y=\lambda y
$$

with boundary conditions

$$
\begin{gathered}
\left(\tilde{a}_{1} \lambda+\tilde{b}_{1}\right) y(0, \lambda)-\left(\tilde{c}_{1} \lambda+\tilde{d}_{1}\right) y^{\prime}(0, \lambda)=0, \\
\left(\tilde{a}_{2} \lambda+\tilde{b}_{2}\right) y(\pi, \lambda)-\left(\tilde{c}_{2} \lambda+\tilde{d}_{2}\right) y^{\prime}(\pi, \lambda)=0,
\end{gathered}
$$

where $\tilde{a}_{k}, \tilde{b}_{k}, \tilde{c}_{k}, \tilde{d}_{k}, \in \mathbf{R},(-1)^{k} \tilde{\delta}_{k}=\tilde{a}_{k} \tilde{d}_{k}-\tilde{b}_{k} \tilde{c}_{k}<0(k=1,2), \tilde{q}(x)$ is a real-valued function in $L^{2}[0, \pi]$.

By virtue of [10], we obtain the following Lemma 2.1, which plays an important role in the proof of Theorem 2.2.

Lemma 2.1. ([10, Theorem]) Let $\left\{\lambda_{n}\right\}(n \geq 0)$ be real and simple spectrum of the Sturm-Liouville problem (1.1)-(1.3) and $\left\{\tilde{\lambda}_{n}\right\}(n \geq 0)$ be real and simple spectrum of the Sturm-Liouville problem (2.1), (2.2), (1.3), respectively and $q-\tilde{q} \in W_{2}^{6}[0, \pi]$. If $\lambda_{n}=\tilde{\lambda}_{n}(n \geq 0)$ and $q(x)=\tilde{q}(x)$ on $[\pi / 2, \pi]$, then

$$
q(x)=\tilde{q}(x) \text { a.e. on }[0, \pi]
$$

and

$$
\frac{\tilde{a}_{1} \lambda+\tilde{b}_{1}}{\tilde{c}_{1} \lambda+\tilde{d}_{1}}=\frac{a_{1} \lambda+b_{1}}{c_{1} \lambda+d_{1}}, \forall \lambda \in \mathbf{C},
$$

where $q(x), \tilde{q}(x)$ are real-valued functions in $L^{2}[0, \pi]$. 
Next, we present the main results in this paper. When $b=\pi / 2$, we get the following uniqueness Theorem 2.2.

Theorem 2.2. Let $\left\{\lambda_{n}\right\}$ and $\left\{\tilde{\lambda}_{n}\right\}$ be a spectrum of both Sturm-Liouville problem (1.1)-(1.3) and Sturm-Liouville problem (2.1)-(2.3), respectively and $q-\tilde{q} \in W_{2}^{6}[0, \pi]$. Iffor any $n(n=0,1,2, \ldots)$

$$
\lambda_{n}=\tilde{\lambda}_{n} \text { and } \frac{y_{n}^{\prime}\left(\pi / 2, \lambda_{n}\right)}{y_{n}\left(\pi / 2, \lambda_{n}\right)}=\frac{\tilde{y}_{n}^{\prime}\left(\pi / 2, \tilde{\lambda}_{n}\right)}{\tilde{y}_{n}\left(\pi / 2, \tilde{\lambda}_{n}\right)},
$$

then

$$
q(x)=\tilde{q}(x) \quad \text { a.e. on }[0, \pi]
$$

and

$$
\frac{\tilde{a}_{1} \lambda+\tilde{b}_{1}}{\tilde{c}_{1} \lambda+\tilde{d}_{1}}=\frac{a_{1} \lambda+b_{1}}{c_{1} \lambda+d_{1}} \text { and } \frac{\tilde{a}_{2} \lambda+\tilde{b}_{2}}{\tilde{c}_{2} \lambda+\tilde{d}_{2}}=\frac{a_{2} \lambda+b_{2}}{c_{2} \lambda+d_{2}}(\forall \lambda \in \mathbf{C}),
$$

where $y_{n}\left(x, \lambda_{n}\right)$ is an eigenfunction of $\lambda_{n}$ and $\tilde{y}_{n}\left(x, \tilde{\lambda}_{n}\right)$ is an eigenfunction of $\tilde{\lambda}_{n}$.

When $b \neq \pi / 2$, the uniqueness theorem of $q(x)$ can be obtained from a part of the second spectrum and some information on eigenfunctions at the point $b \in(0, \pi)$.

Let $m(n)$ be a subsequence of natural numbers such that

$$
m(n)=\frac{n}{\sigma}\left(1+\varepsilon_{n}\right), 0<\sigma \leq \pi, \varepsilon_{n} \rightarrow 0 .
$$

Lemma 2.3. Let $m(n)$ be a subsequence of natural numbers satisfying $(2.5), b \in(0, \pi / 2)$ be such that $\sigma>\frac{2 b}{\pi}$ and $q-\tilde{q} \in W_{2}^{6}[0, \pi]$. If for any $n(n=0,1,2, \ldots)$,

$$
\lambda_{m(n)}=\tilde{\lambda}_{m(n)} \text { and } \frac{y_{m(n)}^{\prime}\left(b, \lambda_{m(n)}\right)}{y_{m(n)}\left(b, \lambda_{m(n)}\right)}=\frac{\tilde{y}_{m(n)}^{\prime}\left(b, \tilde{\lambda}_{m(n)}\right)}{\tilde{y}_{m(n)}\left(b, \tilde{\lambda}_{m(n)}\right)} \text {, }
$$

then

$$
q(x)=\tilde{q}(x) \quad \text { a.e. on }[0, b] \text { and } \quad \frac{\tilde{a}_{1} \lambda+\tilde{b}_{1}}{\tilde{c}_{1} \lambda+\tilde{d}_{1}}=\frac{a_{1} \lambda+b_{1}}{c_{1} \lambda+d_{1}}(\forall \lambda \in \mathbf{C}) .
$$

Let $l(n)$ and $r(n)$ be a subsequence of natural numbers such that

$$
\begin{aligned}
& l(n)=\frac{n}{\sigma_{1}}\left(1+\varepsilon_{1, n}\right), 0<\sigma_{1} \leq \pi, \varepsilon_{1, n} \rightarrow 0, \\
& r(n)=\frac{n}{\sigma_{2}}\left(1+\varepsilon_{2, n}\right), 0<\sigma_{2} \leq \pi, \varepsilon_{2, n} \rightarrow 0
\end{aligned}
$$

and let $\mu_{n}$ be the eigenvalues of the problem (1.1),(1.2) and (2.9) and $\tilde{\mu}_{n}$ be the eigenvalues of the problem (2.1), (2.2) and (2.9).

$$
\left(a_{3} \lambda+b_{3}\right) y(\pi, \lambda)-\left(c_{3} \lambda+d_{3}\right) y^{\prime}(\pi, \lambda)=0,
$$

where $\delta_{3}=a_{3} d_{3}-b_{3} c_{3}<0, \delta_{3} \neq \delta_{2}$.

Using Mochizuki and Trooshin's method, from Lemma 2.3 and Theorem 2.2, we will prove that the following Theorem 2.4 holds. 
Theorem 2.4. Let $l(n)$ and $r(n)$ be subsequence of natural numbers satisfying (2.7) and (2.8), respectively, and $b \in(\pi / 2, \pi)$ be such that $\sigma_{1}>\frac{2 b}{\pi}-1, \sigma_{2}>2-\frac{2 b}{\pi}$ and $q-\tilde{q} \in W_{2}^{6}[0, \pi]$. If for any $n(n=0,1,2, \ldots)$,

$$
\lambda_{n}=\tilde{\lambda}_{n}, \mu_{l(n)}=\tilde{\mu}_{l(n)} \text { and } \frac{y_{r(n)}^{\prime}\left(b, \lambda_{r(n)}\right)}{y_{r(n)}\left(b, \lambda_{r(n)}\right)}=\frac{\tilde{y}_{r(n)}^{\prime}\left(b, \tilde{\lambda}_{r(n)}\right)}{\tilde{y}_{r(n)}\left(b, \tilde{\lambda}_{r(n)}\right)},
$$

then

$$
q(x)=\tilde{q}(x) \text { a.e. on }[0, \pi]
$$

and

$$
\frac{\tilde{a}_{1} \lambda+\tilde{b}_{1}}{\tilde{c}_{1} \lambda+\tilde{d}_{1}}=\frac{a_{1} \lambda+b_{1}}{c_{1} \lambda+d_{1}} \text { and } \frac{\tilde{a}_{2} \lambda+\tilde{b}_{2}}{\tilde{c}_{2} \lambda+\tilde{d}_{2}}=\frac{a_{2} \lambda+b_{2}}{c_{2} \lambda+d_{2}}(\forall \lambda \in \mathbf{C})
$$

\section{Proofs of main results}

In this section, we present the proofs of main results in this paper.

Proof of Theorem 2.2. We give the proof of Theorem 2.2 by two steps.

Step 1: From the assumptions of Theorem 2.2, similar to the proof of Lemma 2.1, we have

$$
q(x)=\tilde{q}(x) \quad \text { a.e. on }[0, \pi / 2] \text { and } \quad \frac{\tilde{a}_{1} \lambda+\tilde{b}_{1}}{\tilde{c}_{1} \lambda+\tilde{d}_{1}}=\frac{a_{1} \lambda+b_{1}}{c_{1} \lambda+d_{1}}(\forall \lambda \in \mathbf{C}) \text {. }
$$

Step 2: Consider the following supplementary problem

$$
\begin{aligned}
& L_{1} y=-y^{\prime \prime}+q_{1}(x) y=\lambda y, \\
& q_{1}(x)=q(\pi-x), x \in[0, \pi],
\end{aligned}
$$

with the boundary conditions

$$
\begin{gathered}
\left(a_{2} \lambda+b_{2}\right) y(0, \lambda)-\left(c_{2} \lambda+d_{2}\right) y^{\prime}(0, \lambda)=0 \\
\left(a_{1} \lambda+b_{1}\right) y(\pi, \lambda)-\left(c_{1} \lambda+d_{1}\right) y^{\prime}(\pi, \lambda)=0 .
\end{gathered}
$$

and

$$
\begin{aligned}
& \tilde{L}_{1} y=-y^{\prime \prime}+\tilde{q}_{1}(x) y=\lambda y, \\
& \tilde{q}_{1}(x)=\tilde{q}(\pi-x), x \in[0, \pi],
\end{aligned}
$$

with the boundary conditions

$$
\begin{gathered}
\left(\tilde{a}_{2} \lambda+\tilde{b}_{2}\right) y(0, \lambda)-\left(\tilde{c}_{2} \lambda+\tilde{d}_{2}\right) y^{\prime}(0, \lambda)=0 \\
\left(\tilde{a}_{1} \lambda+\tilde{b}_{1}\right) y(\pi, \lambda)-\left(\tilde{c}_{1} \lambda+\tilde{d}_{1}\right) y^{\prime}(\pi, \lambda)=0 .
\end{gathered}
$$


Repeating the Step 1 for the supplementary problem, we get

$$
q(x)=\tilde{q}(x) \quad \text { a.e. on }[\pi / 2, \pi] \text { and } \quad \frac{\tilde{a}_{2} \lambda+\tilde{b}_{2}}{\tilde{c}_{2} \lambda+\tilde{d}_{2}}=\frac{a_{2} \lambda+b_{2}}{c_{2} \lambda+d_{2}}(\forall \lambda \in \mathbf{C}) .
$$

By virtue of (3.1) and (3.8), this yields

$$
q(x)=\tilde{q}(x) \quad \text { a.e. on } \quad[0, \pi]
$$

and

$$
\frac{\tilde{a}_{1} \lambda+\tilde{b}_{1}}{\tilde{c}_{1} \lambda+\tilde{d}_{1}}=\frac{a_{1} \lambda+b_{1}}{c_{1} \lambda+d_{1}} \text { and } \frac{\tilde{a}_{2} \lambda+\tilde{b}_{2}}{\tilde{c}_{2} \lambda+\tilde{d}_{2}}=\frac{a_{2} \lambda+b_{2}}{c_{2} \lambda+d_{2}}(\forall \lambda \in \mathbf{C}) .
$$

Therefore, Theorem 2.2 is proved.

Next, we show that Lemma 2.3 holds.

Proof of Lemma 2.3. Multiplying (2.1) by $y$, (1.1) by $\tilde{y}$, subtracting and integrating from 0 to b, we obtain

$$
\begin{aligned}
G(\lambda) & :=\int_{0}^{b} Q(x) y(x, \lambda) \tilde{y}(x, \lambda) d x \\
& =\left.\left[\tilde{y}(x, \lambda) y^{\prime}(x, \lambda)-y(x, \lambda) \tilde{y}^{\prime}(x, \lambda)\right]\right|_{0} ^{b} .
\end{aligned}
$$

From (1.2), (2.2) and the assumption

$$
\frac{y_{m(n)}^{\prime}\left(b, \lambda_{m(n)}\right)}{y_{m(n)}\left(b, \lambda_{m(n)}\right)}=\frac{\tilde{y}_{m(n)}^{\prime}\left(b, \tilde{\lambda}_{m(n)}\right)}{\tilde{y}_{m(n)}\left(b, \tilde{\lambda}_{m(n)}\right)},
$$

we get

$$
G\left(\lambda_{m}(n)\right)=0, n \in N .
$$

Next, we will show $G(\lambda)=0, \forall \lambda \in \mathbf{C}$.

From (3.9), we see that the entire function $G(\lambda)$ is a function of exponential type $\leq 2 b$ and we have

$$
|G(\lambda)| \leq M e^{2 b r|\sin \theta|},
$$

where $M$ is a positive constant, $\lambda=r e^{i \theta}$.

Define the indicator of function $G(\lambda)$ by

$$
h(\theta)=\limsup _{\lambda \rightarrow+\infty} \frac{\ln \left|G\left(r e^{i \theta}\right)\right|}{r} .
$$

Since $|\operatorname{Im} \lambda|=r|\sin \theta|, \theta=\arg \lambda$, from (3.10) and (3.11), we get

$$
h(\theta) \leq 2 b|\sin \theta| .
$$

Let $n(r)$ be the number of zeros of $G(\lambda)$ in the disk $|\lambda| \leq r$. From the assumption of Lemma 2.3 and the asymptotic form (1.4) of the eigenvalues $\lambda_{n}$, we obtain

$$
n(r) \geq 2 \sum_{\frac{n}{\sigma}[1+O(1 / n)]<r} 1 \geq 2 \sigma r[1+\varepsilon(r)], r \rightarrow \infty,
$$


where $\varepsilon(r) \rightarrow 0, r \rightarrow \infty,[x]$ is the integer part of $x$.

For the case $\sigma>\frac{2 b}{\pi}$,

$$
\liminf _{n \rightarrow \infty} \frac{n(r)}{r} \geq 2 \sigma>\frac{4 b}{\pi} \geq \frac{1}{2 \pi} \int_{0}^{2 \pi} h(\theta) d \theta .
$$

According to [19], for any entire function $G(\lambda)$ of exponential type, not identically zero, then

$$
\liminf _{n \rightarrow \infty} \frac{n(r)}{r} \leq \frac{1}{2 \pi} \int_{0}^{2 \pi} h(\theta) d \theta .
$$

The inequalities (3.14) and (3.15) imply that $G(\lambda)=0, \forall \lambda \in \mathbf{C}$.

Similar to the proof of the Lemma 2.1, we have

$$
q(x)=\tilde{q}(x) \quad \text { a.e. on }[0, b] \text { and } \quad \frac{\tilde{a}_{1} \lambda+\tilde{b}_{1}}{\tilde{c}_{1} \lambda+\tilde{d}_{1}}=\frac{a_{1} \lambda+b_{1}}{c_{1} \lambda+d_{1}} .
$$

This completes the proof of Lemma 2.3.

Now, we prove that Theorem 2.4 is valid.

Proof of Theorem 2.4. From

$$
\lambda_{r(n)}=\tilde{\lambda}_{r(n)}, \text { and } \frac{y_{r(n)}^{\prime}\left(b, \lambda_{r(n)}\right)}{y_{r(n)}\left(b, \lambda_{r(n)}\right)}=\frac{\tilde{y}_{r(n)}^{\prime}\left(b, \tilde{\lambda}_{r(n)}\right)}{\tilde{y}_{r(n)}\left(b, \tilde{\lambda}_{r(n)}\right)},
$$

where $r(n)$ satisfies (2.8) and $\sigma_{2}>2-\frac{2 b}{\pi}$, according to Lemma 2.3, we get

$$
q(x)=\tilde{q}(x), \quad \text { a.e. on }[b, \pi] \text { and } \quad \frac{\tilde{a}_{2} \lambda+\tilde{b}_{2}}{\tilde{c}_{2} \lambda+\tilde{d}_{2}}=\frac{a_{2} \lambda+b_{2}}{c_{2} \lambda+d_{2}}(\forall \lambda \in \mathbf{C})
$$

Let $y_{n}\left(x, \lambda_{n}\right)$ and $\tilde{y}_{n}\left(x, \lambda_{n}\right)$ be the eigenfunctions of Sturm-Liouville problems (1.1)-(1.3) and (2.1)-(2.3), corresponding to eigenvalue $\lambda_{n}$, respectively. Since the eigenfunctions $y_{n}\left(x, \lambda_{n}\right)$ and $\tilde{y}_{n}\left(x, \lambda_{n}\right)$ satisfy the same boundary condition at $\pi$ and $\tilde{q}(x)=q(x)$, a.e. on $[b, \pi]$, we obtain

$$
y_{n}\left(x, \lambda_{n}\right)=\alpha_{n} \tilde{y}_{n}\left(x, \lambda_{n}\right), x \in[b, \pi], n \in \mathbf{N}
$$

where $\alpha_{n}$ are constants.

From (3.9), (3.17), (1.2) and (2.2), we obtain

$$
G\left(\lambda_{n}\right)=0, n \in \mathbf{N}
$$

and

$$
G\left(\mu_{l(n)}\right)=0, n \in \mathbf{N},
$$

where $\lambda_{n}$ and $\mu_{l(n)}$ satisfy (1.4). 
Let us count the number of $\lambda_{n}$ and $\mu_{l(n)}$ located inside the disc of radius $r$ (sufficiently large $r$ ). We see that there are $1+2 r[1+o(1)]$ of $\lambda_{n}$ and $1+2 r \sigma_{1}[1+o(1)]$ of $\mu_{l(n)}$ located inside the disc of radius $r$. Therefore

$$
n(r)=2+2 r\left[1+\sigma_{1}+o(1)\right]
$$

Hence

$$
\lim _{n \rightarrow \infty} \frac{n(r)}{r}=2\left(\sigma_{1}+1\right) .
$$

Considering the condition $\sigma_{1}>\frac{2 b}{\pi}-1$, we get

$$
\lim _{n \rightarrow \infty} \frac{n(r)}{r} \geq 2 \sigma_{1}>\frac{4 b}{\pi} \geq \frac{1}{2 \pi} \int_{0}^{2 \pi} h(\theta) d \theta
$$

According to [19], for any entire function $G(\lambda)$ of exponential type, not identically zero, we see the following inequality (3.19) holds.

$$
\liminf _{n \rightarrow \infty} \frac{n(r)}{r} \leq \frac{1}{2 \pi} \int_{0}^{2 \pi} h(\theta) d \theta .
$$

The inequalities (3.18) and (3.19) imply that

$$
G(\lambda)=0, \forall \lambda \in \mathbf{C} .
$$

From (3.20), we can show that

$$
q(x)=\tilde{q}(x) \quad \text { a.e. on }[0, b] \text { and } \quad \frac{\tilde{a}_{1} \lambda+\tilde{b}_{1}}{\tilde{c}_{1} \lambda+\tilde{d}_{1}}=\frac{a_{1} \lambda+b_{1}}{c_{1} \lambda+d_{1}} .
$$

From (3.15) and (3.21), we have

$$
q(x)=\tilde{q}(x) \quad \text { a.e. on }[0, \pi]
$$

and

$$
\frac{\tilde{a}_{1} \lambda+\tilde{b}_{1}}{\tilde{c}_{1} \lambda+\tilde{d}_{1}}=\frac{a_{1} \lambda+b_{1}}{c_{1} \lambda+d_{1}} \text { and } \frac{\tilde{a}_{2} \lambda+\tilde{b}_{2}}{\tilde{c}_{2} \lambda+\tilde{d}_{2}}=\frac{a_{2} \lambda+b_{2}}{c_{2} \lambda+d_{2}}(\forall \lambda \in \mathbf{C}) .
$$

Hence, the proof of Theorem 2.4 is completed.

\section{Acknowledgements}

The author acknowledges helpful comments and suggestions from the referees. 


\section{References}

[1] V. A. Ambartsumyan, Über eine frage der eigenwerttheorie, Zeitschrift für Physik., 53 (1929), 690-695.

[2] W. Feller, The parabolic differential equations and the associated semi-groups of transforms, Ann. of Math., 55(1952), 468-519.

[3] R. E. Gaskell, A problem in heat conduction and an expansion theorem, Amer. J. Math., 64(1942), 447-455.

[4] W. F. Bauer, Modified Sturm-Liouville systems, Quart. Appl. Math., 11(1953), 273-282.

[5] R. L. Peek, Jr., Solutions to a problem in diffusion employing a non-orthogonal sine series, Ann of Math. 30 (1929), 265-269.

[6] C. T. Fulton, Two-point boundary value problems with eigenvalue parameter contained in the boundary conditions, Proc. Roy. Soc. Edinburgh 77a(1977), 293-308.

[7] P. A. Binding, P. J. Browne and K. Seddighi, Sturm-Liouville problems with eigenparameter dependent boundary conditions, Proc. Roy. Soc. Edinburgh., 37(1993), 57-72.

[8] P. J. Browne and B. D. Sleeman, Inverse nodal problems for Sturm-Liouville equations with eigenparameter dependent boundary conditions, Inverse Problems, 12 (1996), 377-381.

[9] N. J. Guliyev, The regularized trace formula for the Sturm-Liouville equation with spectral parameter in the boundary conditions, Proc. Inst. Math. Natl. Alad. Sci. Azerb., 22(2005), 99-102.

[10] Y. P. Wang, C. F. Yang and Z. Y. Huang, Half inverse problem for the Sturm-Liouville operator with eigenparameter dependent boundary conditions, Submitted to Turkish Journal of Mathematics. 2010. 08.

[11] H. Hochstadt and B. Lieberman, An inverse Sturm-Liouville problem wity mixed given data, SIAM Journal of Applied Mathematics, 34(1978), 676-680.

[12] K. Mochizuki and I. Trooshin, Inverse problem for interior spectral data of Sturm-Liouville operator, J. Inverse Ill-Posed Problems 9 (2001), 425-433.

[13] C. F. Yang and X. P. Yang, An interior inverse problem for the Sturm-Liouville operator with discontinuous conditions, Applied Mathematics Letters, 22(2009), 1315-1319.

[14] C. Willis, Inverse Sturm-Liouville problems with two discontinuities, Inverse Problems, 1 (1985), $263-289$.

[15] C. T. Shieh and V. A. Yurko, Inverse nodal and inverse spectral problems for discontinuous boundary value problems, Journal of Mathematical Analysis and Applications, 347 (1) (2008), 266-272.

[16] C. K. Law and J. Tsay, On the well-posedness of the inverse nodal problem, Inverse Problems, 17 (2001), 14931512.

[17] C. L. Shen and C. T. Shieh, An inverse nodal problem for vectorial Sturm-Liouville equation, Inverse Problems, 16 (2000), 349-56.

[18] V. A. Yurko, Inverse spectral problems for Sturm-Liouville differential operators on a finite interval, J. Inv. IllPosed Problems, 17 (2009), 639-694.

[19] B. J. Levin, Distribution of Zeros of Entire Functions, AMS. Transl. Vol. 5, Providence, 1964.

[20] V. A. Yurko, Method of Spectral Mappings in the Inverse Problem Theory. Inverse Ill-posed Problems Ser., VSP, Utrecht, 2002.

[21] B. M. Levitan and I. S. Sargsjan, Sturm-Liouville and Dirac operators. Kluwer Academic Publishers, 1990.

[22] N. Dunford and J. T. Schwarz, Linear Operators (Part II). Interscience Publishers, 1963.

Department of Applied Mathematics, Nanjing Forestry University, Nanjing, 210037, Jiangsu, China.

E-mail: ypwang@njfu.com.cn 